PROCEEDINGS OF THE

AMERICAN MATHEMATICAL SOCIETY

Volume 127, Number 12, Pages 3525-3526

S 0002-9939(99)05431-3

Article electronically published on July 27, 1999

\title{
AN ANALYTIC INVARIANT OF ORDINARY CURVE SINGULARITIES
}

\author{
JOAQUIM ROÉ
}

(Communicated by Ron Donagi)

\begin{abstract}
It is well known that analytically equivalent ordinary plane curve singularities have projectively equivalent tangent cones. In this note we introduce an analytic invariant in order to show two non analytically equivalent ordinary 5 -fold points with projectively equivalent (or equal) tangent cones.
\end{abstract}

Let $\mathcal{C}$ be a plane curve, having an ordinary singularity of multiplicity 5 at the point $O$. Choose analytic local coordinates $x, y$ in a neighborhood of $O$ and analytic parametrisations $\gamma_{i}(t)=\left(x_{i}(t), y_{i}(t)\right)$ of the branches of the curve, with $\gamma_{i}(0)=O$, $\gamma_{i}^{\prime}(0) \neq 0$ for $i=1,2,3,4,5$. We fix the following notations:

$$
\begin{aligned}
a_{i} & =\frac{d x_{i}}{d t}(0), \quad b_{i}=\frac{d y_{i}}{d t}(0), \\
\kappa_{i} & =b_{i} \frac{d^{2} x_{i}}{d t^{2}}(0)-a_{i} \frac{d^{2} y_{i}}{d t^{2}}(0) .
\end{aligned}
$$

We could say that $b_{i} x-a_{i} y=0$ is the tangent line at $O$ to the $i$-th branch and $\kappa_{i}$ its curvature, were it not for the fact that the words line and curvature have no meaning in an analytic setting. Nevertheless we do have the following.

Proposition. The expression

$$
\mathcal{I}=\operatorname{det}\left(\begin{array}{ccccc}
\kappa_{1} & a_{1}^{3} & a_{1}^{2} b_{1} & a_{1} b_{1}^{2} & b_{1}^{3} \\
\kappa_{2} & a_{2}^{3} & a_{2}^{2} b_{2} & a_{2} b_{2}^{2} & b_{2}^{3} \\
\kappa_{3} & a_{3}^{3} & a_{3}^{2} b_{3} & a_{3} b_{3}^{2} & b_{3}^{3} \\
\kappa_{4} & a_{4}^{3} & a_{4}^{2} b_{4} & a_{4} b_{4}^{2} & b_{4}^{3} \\
\kappa_{5} & a_{5}^{3} & a_{5}^{2} b_{5} & a_{5} b_{5}^{2} & b_{5}^{3}
\end{array}\right)
$$

is an analytic relative invariant of $\mathcal{C}$, i.e. its vanishing does not depend on the analytic coordinates or parametrisation chosen, nor is it affected by the action of the analytic group on the plane.

Proof. Let us consider the scroll $S_{1,4} \subset \mathbf{P}^{6}$ which Semple showed in [3] to be the variety that contains the points infinitely near $O$ in its second neighborhood.

$$
S_{1,4}=\left\{\left[z_{0}, z_{1}, \ldots, z_{6}\right] \in \mathbf{P}^{6} \mid \operatorname{rank}\left(\begin{array}{ccccc}
z_{0} & z_{2} & z_{3} & z_{4} & z_{5} \\
z_{1} & z_{3} & z_{4} & z_{5} & z_{6}
\end{array}\right)=1\right\}
$$

Received by the editors March 2, 1998.

1991 Mathematics Subject Classification. Primary 14H20.

The author was partially supported by 1997FI-00141, CAICYT PB95-0274 and "AGEAlgebraic Geometry in Europe" contract no. ERB940557. 
Each one of our five branches has a single point in the second neighborhood of $O$. This point is

$$
p_{i}=\left(\kappa_{i} a_{i}, \kappa_{i} b_{i}, a_{i}^{4}, a_{i}^{3} b_{i}, a_{i}^{2} b_{i}^{2}, a_{i} b_{i}^{3}, b_{i}^{4}\right) \in S_{1,4} .
$$

Semple also proved that transformations of the plane which are analytic at $O$ and leave $O$ invariant induce self-collineations on $S_{1,4}$. This reduces the problem to seeing that $\mathcal{I}$ is a projective invariant of $\left\{p_{1}, \ldots, p_{5}\right\}$.

It is well known that in $S_{1,4}$ there is a unique family of rational normal quartic curves cut out by the 4 -planes

$$
\Pi_{\alpha}:\left\{\begin{array}{l}
\alpha_{0} z_{0}+\alpha_{1} z_{2}+\alpha_{2} z_{3}+\alpha_{3} z_{4}+\alpha_{4} z_{5}=0 \\
\alpha_{0} z_{1}+\alpha_{1} z_{3}+\alpha_{2} z_{4}+\alpha_{3} z_{5}+\alpha_{4} z_{6}=0
\end{array}\right.
$$

with $\alpha=\left[\alpha_{0}, \ldots, \alpha_{4}\right] \in \mathbf{P}^{4}$ (see [2] for generalities on rational normal scrolls). Substituting the coordinates of the points, we find that $p_{i} \in \Pi_{\alpha}$ if and only if

$$
\omega_{i}(\alpha)=\alpha_{0} \kappa_{i}+\alpha_{1} a_{i}^{3}+\alpha_{2} a_{i}^{2} b_{i}+\alpha_{3} a_{i} b_{i}^{2}+\alpha_{4} b_{i}^{3}=0 .
$$

Thus there exists a single $\Pi_{\alpha}$ containing the five points if and only if the equations $\omega_{i}(\alpha)=0, i=1, \ldots, 5$, have a common solution, that is, if the determinant $\mathcal{I}$ vanishes. As the property of the five points belonging to one of the rational normal quartics is clearly projectively invariant, the proof is complete.

Remark 1. If $\kappa_{i}=0 \forall i$ (that is, if $O$ is an inflection point of every branch), the invariant is obviously zero. Consequently, if for a curve $C$ the invariant is nonzero, no analytic transformation can make its five branches into inflections. Although curvature has no analytic meaning in a smooth point of a curve, it is meaningful to say whether five smooth branches through a point are "curved with respect to one another" or not, and this is told by the invariant $\mathcal{I}$.

Remark 2. Looking at the proof, we can say further that any number of branches whose points in the second neighborhood lie on a rational normal quartic of $S_{1,4}$ are not curved with respect to one another. One may also note that given 4 or less smooth branches with distinct tangent directions there is a $\Pi_{\alpha}$ containing their points of the second neighborhood, so five is the minimum number for which such an invariant is possible.

Example. The singularities $\left(x^{4}-y^{4}\right) y=0$ and $\left(x^{4}-y^{4}\right)\left(y-x^{2}\right)=0$ are not analytically isomorphic.

\section{REFERENCES}

[1] Casas-Alvero, E., Singularities of plane curves (1997), to appear.

[2] Harris, J., Algebraic Geometry, GTM 133, Springer Verlag 1992. MR 93j:14001

[3] Semple, J.G., Some investigations in the geometry of curve and surface elements, Proc. London Math. Soc. (3) 4 (1954), 24-49. MR 15:820c

Departament d’Àlgebra i Geometria, Universitat de Barcelona Gran Via, 585, E08007, Barcelona, Spain

E-mail address: jroevell@cerber.mat.ub.es 